\section{Similarity and attribution in concept combination: Reply to Wisniewski}

\author{
ZACHARY ESTES and SAM GLUCKSBERG \\ Princeton University, Princeton, New Jersey
}

Wisniewski raises several important points in his comment on our study of the (non)role of similarity in concept combination. He notes that feature diagnosticity is an important factor in property attribution. He also points out that relational interpretations of combined concepts depend on thematic role plausibility, not constituent similarity. We agree with both of these points. Wisniewski further identifies a possible item artifact that might account for our results. However, reexamination of those items fails to support this hypothesis. Wisniewski also claims that an alignment process is necessary to specify how an attributed property should be instantiated in the combined concept. We argue instead that postcomprehension elaboration processes can account for specific property instantiations. Finally, we suggest that the perceived similarity between the constituents of a combined concept is an outcome of the comprehension process, not a prior condition for, or an integral part of, that process.

Wisniewski (2000) raises several important points in his comment on our study of the (non)role of similarity in concept combination. In that paper (Estes \& Glucksberg, 2000), we contrasted two views of property attribution. According to the alignment view, property attribution involves an alignment stage in which alignable differences must first be identified before properties of a modifier can be attributed to a head noun (Wisniewski, 1997). Because alignable differences are more likely to be found for similar than for dissimilar noun concepts (Gentner \& Markman, 1994), constituent similarity should be a facilitating, if not necessary, condition for property attribution. Accordingly, property attribution should be more likely for combined concepts with similar constituents than for combined concepts with dissimilar constituents. In contrast, we argued that property attribution is independent of constituent similarity per se. Instead, property attribution is likely whenever a salient property of the modifier is attributable to a relevant dimension of the head noun. In the example shark lawyer, a salient property (e.g. "vicious") of the modifier fills a relevant dimension (e.g., TEMPERAMENT) of the head noun. The results of our experiment supported this interactive view of property attribution.

We thank Yevgeniya Goldvarg and Mary Newsome for helpful comments. This research was supported by a National Science Foundation graduate research fellowship to Z.E. and by Grant SBR-9712601 from the National Science Foundation to Princeton University (S.G., principal investigator). Correspondence should be addressed to Z. Estes, Department of Psychology, Green Hall, Princeton University, Princeton, NJ 08544-1010 (e-mail: zcestes@princeton.edu).
Wisniewski raises a number of issues, which we will address in turn.

The role of diagnosticity. Wisniewski states that "the process involved in producing property interpretations is generally facilitated by constituent similarity" (2000, p. 37). But our results suggest that constituent similarity is independent of property attribution. Feature diagnosticity, Wisniewski adds, is also important. Here we are in agreement. As we put it in our original paper, our "notion of salience subsumes diagnosticity as one potential determinant of which property to attribute" (Estes \& Glucksberg, 2000, p. 32).

The (non)role of similarity in relation interpretation. Wisniewski notes that relation interpretation depends on thematic role plausibility, not constituent similarity. Again we agree. Since our paper concerned property attribution, any implications about relation interpretation were purely statistical. That is, most combined concepts are interpreted by means of either relations or property attribution. ${ }^{\prime}$ Thus, if there are few property interpretations for a given combined concept, there must be many relation interpretations.

A possible item artifact. Wisniewski correctly points out that if a combination does not have a plausible relation, it will, perforce, be interpreted by means of property attribution. Perhaps the prevalence of property interpretation in our high-salience high-relevance $(\mathrm{HH})$ condition was due to the items in this condition not having plausible relations, he suggests. However, reexamination of those items fails to support this hypothesis. Fourteen of the $20 \mathrm{HH}$ items not only had plausible relations, they were actually interpreted relationally by some participants. ${ }^{2}$ If the prevalence of property attribution in the $\mathrm{HH}$ condition was due to the 6 items that may not have had plausible relations, then removing these items from analyses should delete the effect. Instead, the results for the 14 items that did have plausible relations are nearly identical to the results based on all 20 items. The mean proportions of property attribution for the subset of 14 items were $.74, .21$, and .18 for the $\mathrm{HH}, \mathrm{HL}$, and $\mathrm{LH}$ conditions, respectively. We conclude that the differences in property attribution were due to the degree to which they satisfied the conditions for property attribution-that is, salient properties of the modifier matching relevant dimensions of the head noun.

The role of alignment in instantiating attributed properties. In addition to finding alignable differences, Wisniewski claims that the alignment process is also necessary to specify how an attributed property should be instantiated. Taking zebra clam as an example, people can determine the size and location of the stripes by aligning "the body of a zebra with the shell of the clam and the exterior surface of the zebra with the exterior surface of the clam" (p. 38). Although this hypothesis may be tenable, it certainly is not necessary. Properties such as "stripes" 
may simply be left unspecified in the interpretation "striped clam." Then, if specification were to become necessary, general world knowledge would suffice. Stripes are usually on an exterior surface, and their size can be adjusted to fit their object. And just as zebra fish have different colored stripes from those of zebras (i.e., zebra fish usually have blue-green and yellow stripes), zebra clams could have different colored stripes as well. Clearly, alignment alone cannot produce such novel property instantiations. Instead, they must be determined by postcomprehension elaboration processes (cf. Murphy, 1988).

Is similarity necessary for property attribution? Wisniewski is clear on this question: "In order to apply a property of one concept to another, the concepts must be similar at least to some degree" $(2000$, p. 36 ; emphasis added). We stress that the grounds for such similarity may well be so abstract as to be meaningless (cf. Goodman, 1972, pp. 437-447). What prior similarities exist between zebra and clam? The only similarity that need exist prior to comprehension is that both zebras and clams have surfaces. We suggest that the perceived similarity between the constituents of a combined concept is an outcome of the comprehension process, not a prior condition for, or an integral part of, that process.

In conclusion, our results suggest that constituent similarity has little to do with property attribution. We hope that Wisniewski's comment and our reply have clarified the issues, and that any remaining differences between the views can be adjudicated by further research.

\section{REFERENCES}

ESTes, Z., \& GlucksBerg, S. (2000). Interactive property attribution in concept combination. Memory \& Cognition, 28, 28-34.

Gentner, D., \& Markman, A. B. (1994). Structural alignment in comparison: No difference without similarity. Psychological Science, $\mathbf{5}$, $152-158$

Goodman, N. (1972). Problems and projects. New York: BobbsMerrill.

MurPhy, G. L. (1988). Comprehending complex concepts. Cognitive Science, 12, 529-562.

WISNIEWSKi, E. J. (1997). When concepts combine. Psychonomic Bulletin \& Review, 4, 167-183.

WISNIEWSKI, E. J. (2000). Similarity, alignment, and conceptual combination: Comment on Estes and Glucksberg. Memory \& Cognition, 28, 35-38.

\section{NOTES}

1. In our experiment, for example, over $96 \%$ of all interpretations were either property or relation interpretations.

2. Indeed, 6 of the 8 items that Wisniewski characterized as lacking plausible relations were in fact interpreted with relations by one or more participants.

(Manuscript received June 22, 1999; accepted for publication July 29, 1999.) 